\title{
Light elements in stars with exoplanets
}

\author{
E. Delgado Mena ${ }^{1}$, M. C. Gálvez-Ortiz ${ }^{2}$, J. I. González-Hernández ${ }^{3}$, \\ G. Israelian ${ }^{1}$, N.C. Santos ${ }^{4},{ }^{5}$, R. Rebolo ${ }^{1},{ }^{6}$ and C. Dominguez \\ Cerdeña ${ }^{1}$ \\ ${ }^{1}$ Instituto de Astrofísica de Canarias, E-38200 La Laguna, Tenerife, Spain. \\ email: edm@iac.es \\ ${ }^{2}$ Centre for Astrophysics Research, Science and Technology Research Institute, University of \\ Hertfordshire, Hatfield AL10 9AB, UK. \\ ${ }^{3}$ Departamento de Astrofísica, Facultad de Ciencias Físicas, Universidad Complutense de \\ Madrid, E-28040, Spain. \\ ${ }^{4}$ Centro de Astrofísica, Universidade do Porto, Rua das Estrelas, 4150-762 Porto, Portugal. \\ ${ }^{5}$ Observatoire de Genève, 51 ch. des Maillettes, CH-1290 Sauverny, Switzerland. \\ ${ }^{6}$ Consejo Superior de Investigaciones Científicas, E-28006, Madrid, Spain.
}

\begin{abstract}
Light elements are important tracers of the internal stellar structure and kinematics. $\mathrm{Li}$ and $\mathrm{Be}$ are both burned in the stellar interiors but Be requires much higher temperatures and thus we can expect to measure Be abundances in stars which have no detectable Li in their atmospheres. The study of these elements can give us information about processes related to the angular momentum history of these stars, since rotation and angular momentum loss are important mechanisms responsible for the depletion of light elements. Additionally, if pollution has played an important role in determining the high-metal content of planet host stars, we would expect to find a similar or even higher increase in the $\mathrm{Li}$ and Be contents. We present $\mathrm{Be}$ and $\mathrm{Li}$ abundances in a sample of 69 stars with planets and 31 stars without known planetary companion, spanning a large range of effective temperatures.
\end{abstract}

Keywords. stars: abudances, stars: fundamental parameters, stars: planetary systems, stars: planetary systems: formation, stars: atmospheres

In Fig. 1 we present the derived Be abundances as a function of effective temperature. Be shows a trend similar to previous works if we exclude the subgiant stars with temperatures below $4900 \mathrm{~K}$. Overall, no clear difference seems to exist between planet hosts and comparison stars. Be abundances decrease from a maximum near Teff $=6100$ $\mathrm{K}$ towards both higher and lower temperatures although there is an object that does not follow this trend. Very little, if any, depletion has occurred for stars of this effective temperature. A similar maximum for the Li abundances is also found at about the same temperature. For higher temperatures the values of $\log \mathrm{N}(\mathrm{Be})$ decrease forming the well-known Be gap for F stars, a feature that has a counterpart for Li. The maximum at $6100 \mathrm{~K}$ may be attributed to Galactic chemical evolution effects, since most of the stars in the temperature interval between 6000 and $6200 \mathrm{~K}$ are particularly metal-rich, and Galactic Be abundances are known to increase with the metallicity (Rebolo et al. 1988, Boesgaard et al. 1999). However, high Be abundances are not observed in low temperature metal-rich stars. The fact that Be abundances decrease at low temperatures can be seen as evidence that Be is burned during the main sequence evolution of these stars but this low abundances are not predicted by models (Pinsonneault et al. 1990).

Be and Li abundances are shown in the right panel of Fig. 1. This figure tells us that overall, and taking into account only dwarfs, there seems to be a correlation between the depletions of $\mathrm{Li}$ and $\mathrm{Be}$, in the sense that stars that have depleted their Be have also 

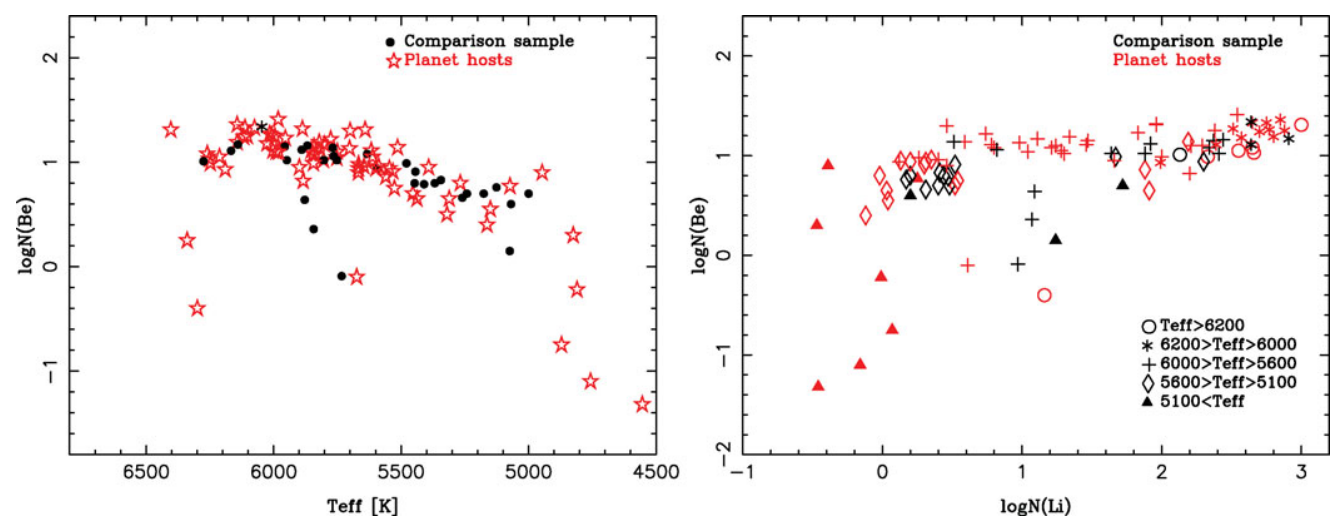

Figure 1. Left panel: Beryllium abundances as a function of effective temperature for stars with (open stars) and without (filled circles) known planets from Santos et al. $(2002,2004)$ and Gálvez Ortiz et al. (2009, submitted). Abundances below log $\mathrm{N}(\mathrm{Be}) \sim 0.8$ are not predicted by models (Pinsonneault et al. 1990) at $\mathrm{T}_{\text {eff }}<5500 \mathrm{~K}$. Right panel: Beryllium abundances as a function of lithium bundances for stars with (red symbols) and without (black symbols) known planets from Gálvez Ortiz et al. (2009, submitted).

strongly depleted their Li. We can see that objects with temperatures above $6000 \mathrm{~K}$ are situated in the upper right corner where there is a maximum of $\mathrm{Li}$ and Be values. In the temperature regime between 5600 and $6000 \mathrm{~K}$ a large dispersion in the $\mathrm{Li}$ abundances is observed while Be remains close to the primordial value. Stars with temperatures below $5600 \mathrm{~K}$ are mostly situated in the left upper-middle panel where both Li and Be are clearly depleted. This temperature seems to mark the onset of strong Be depletion while severe Li depletion begins at $5900 \mathrm{~K}$.

The results presented for Be can be explained in terms of the Galactic chemical evolution. A comparison of the Be abundances of planet hosts and 'single' stars has revealed that, perhaps with a few exceptions, the two samples follow the same behaviour in the $\log \mathrm{N}(\mathrm{Be})$ vs. $\mathrm{T}_{\text {eff }}$ plot. Nevertheless, we have found a small group of stars that present particularly high Be abundances that could signal pollution effects, although other explanations are possible. Measurements of ${ }^{6} \mathrm{Li} /{ }^{7} \mathrm{Li}$ ratio will allow to find signatures of planetary accretion.

\section{References}

Boesgaard, A. M., Deliyannis, C. P., King, J. R. et al. 1999, AJ, 117, 1549

Fischer, D. A. \& Valenti, J. 2005, ApJ, 622, 1102

Gálvez-Ortiz, M.C., Delgado Mena, E., Santos, N.C., Israelian, G. et al. 2009, A $\mathscr{G} A$, submitted Israelian, G., Santos, N. C., Mayor, M., \& Rebolo, R. 2003, A\&A, 405, 753

Israelian, G., Santos, N. C., Mayor, M., \& Rebolo, R. 2004, A\&A, 414, 601

Kurucz, R. L. 1993, CD-ROMs, ATLAS9 Stellar Atmospheres Programs (Cambridge: Smithsonian Astrophys. Obs.)

Pinsonneault, M. H., Kawaler, S. D., \& Demarque, P. 1990, ApJS, 74, 501

Rebolo, R., Abia, C., Beckman, J. E., \& Molaro, P. 1988, A\&\&A, 193, 193

Santos, N. C., Garca Lpez, R. J., Israelian, G., Mayor, M., Rebolo, R., Garca-Gil, A., Prez de Taoro, M. R., \& Randich, S. 2002, A\&A, 386, 1028

Santos, N. C., Israelian, G., Randich, S., Garca Lpez, R. J., \& Rebolo, R. 2004, A\& A, 425, 1013

Santos, N. C., Israelian, G., Garca Lpez, R. J., Mayor, M., Rebolo, R., Randich, S., Ecuvillon, A., \& Domnguez Cerdea, C. 2004, A\&A, 427, 1085

Sousa, S. G., Santos, N. C., Mayor, M., Udry, S., Casagrande, L., Israelian, G., Pepe, F., Queloz, D., Monteiro, M. J. P. F. G. 2008, A\& $A$, 487, 373 